\title{
Erratum to: Saccadic flight strategy facilitates collision avoidance: closed-loop simulation of a cyberfly
}

\author{
Jens Peter Lindemann - Holger Weiss • Ralf Möller • \\ Martin Egelhaaf
}

Published online: 8 March 2012

(c) Springer-Verlag 2012

\section{Erratum to: Biol Cybern (2008) 98:213-227 DOI 10.1007/s00422-007-0205-x}

Due to a parametrisation error in the rendering module of the software, the spatial filtering of the images was done with a smaller filter than stated in the methods section $\left(\sigma \sim 0.4^{\circ}\right.$ instead of $\sigma=2^{\circ}$ ). A correctly parametrised spatial filtering results in quantitative differences in the simulated HSE responses. However, the qualitative results of the simulation can be reproduced after a small modification in the model's peripheral processing layer.

\section{Reproduced results}

Applying spatial low-pass filtering with $\sigma=2^{\circ}$, approximating the filter properties of a blowfly's eye blurs the edges in the simulated retinal image. This blurring leads to slight attenuation and smoothing of the simulated neuronal responses compared to the signals generated from less blurred images. With otherwise unchanged parameters for the other modules, this subtle difference in the signal time courses results in additional wall collisions of the cyberfly when the symmetry-breaking extension of the controller is enabled.

To enhance edge contrast of the retinal images, a weak lateral inhibition was added to the spatio-temporal processing of input images. The inhibition was implemented as a spatial convolution with a $3 \times 3$ mask:

$$
\left(\begin{array}{rrr}
-0.053 & -0.076 & -0.053 \\
-0.076 & 1.515 & -0.076 \\
-0.053 & -0.076 & -0.053
\end{array}\right)
$$

Lateral inhibition between retinotopic elements is a common mechanism for contrast enhancement in biological visual systems and has also been proposed to be relevant for the lamina monopolar cells (LMCs) of the fly (Laughlin and Osorio 1989).

Figures 1-5 show the simulation results of the updated model. These results qualitatively agree with the results shown in Figs. 7-11 of the original publication.

The online version of the original article can be found under doi:10.1007/s00422-007-0205-x.

J. P. Lindemann $(\varangle) \cdot$ H. Weiss $\cdot$ M. Egelhaaf

Neurobiologie, Fakultät für Biologie, Universität Bielefeld,

Postfach 1001 31, 33501 Bielefeld, Germany

e-mail: Jens.Lindemann@Uni-Bielefeld.DE

R. Möller

AG Technische Informatik, Technische Fakultät,

Universität Bielefeld, Postfach 1001 31, 33501 Bielefeld, Germany 


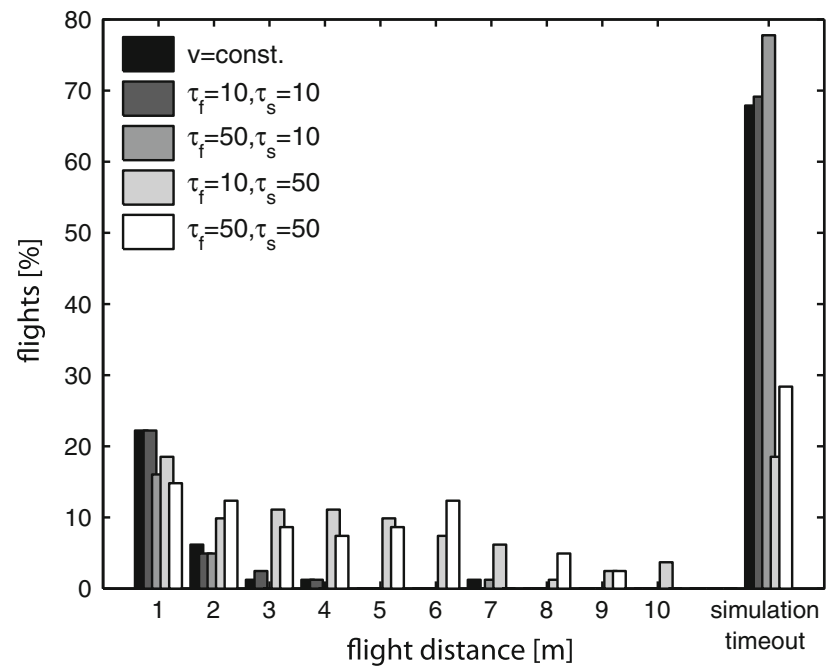

Fig. 1 Histograms showing the distribution of flight lengths in dependence on controller parametrisation. Compare to Fig. 7 in the original paper

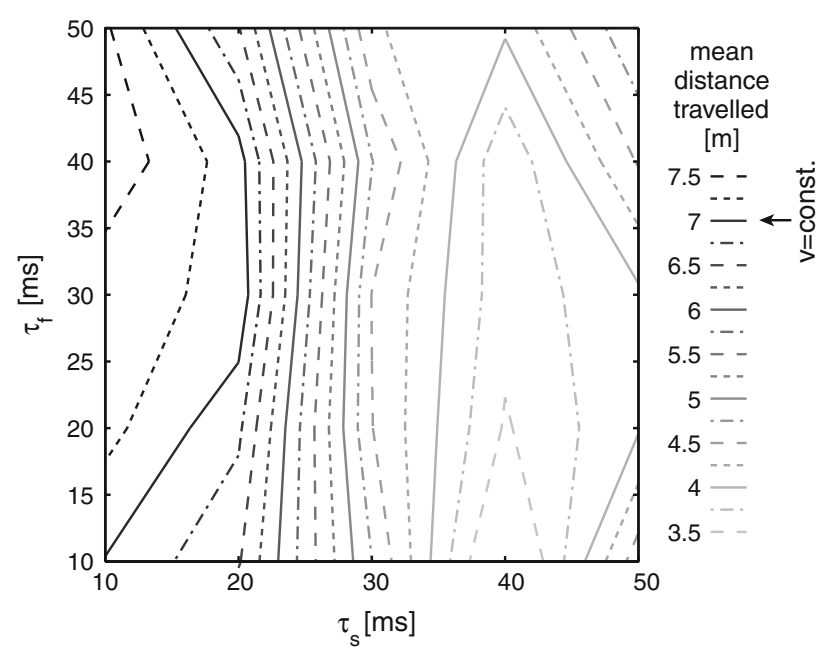

Fig. 2 Dependency of cumulated flight distance on the controller parameters. Compare to Fig. 8 of the original paper
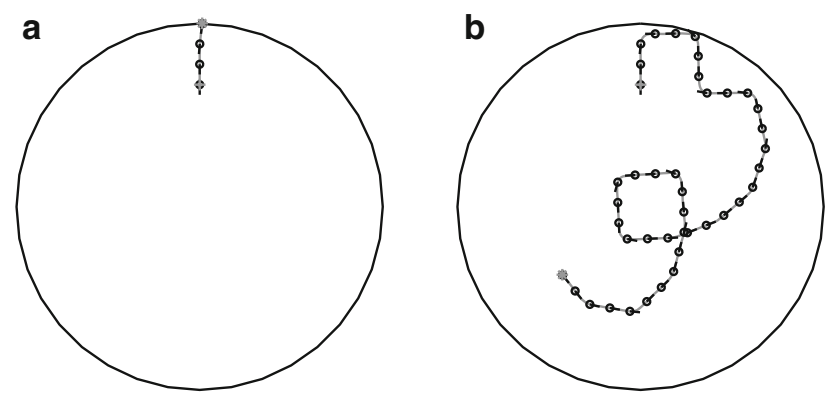

Fig. 3 Two trajectories illustrating the effect of the symmetry breaking extension of the controller. Compare to Fig. 9 of the original paper

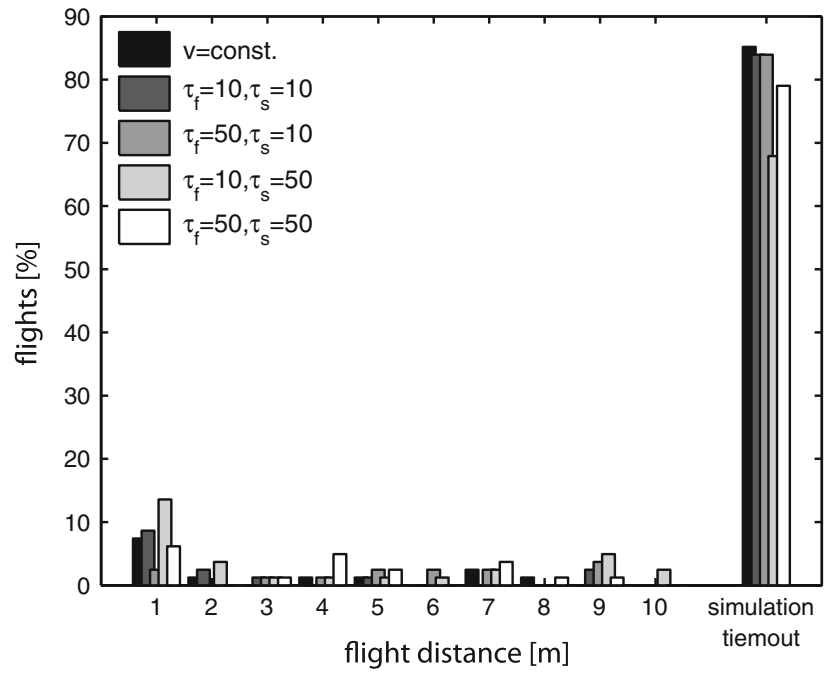

Fig. 4 Histograms of flight lengths for the extended controller. Compare to Fig. 10 of the original paper
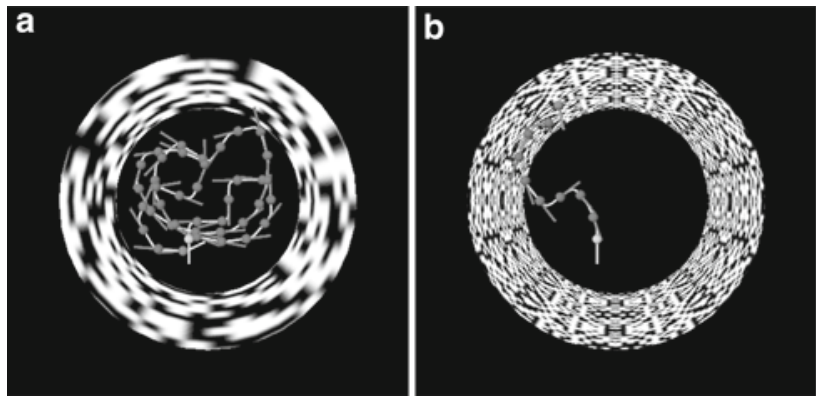

Fig. 5 Texture dependent change in performance. Compare to Fig. 11 of the original paper

\section{Reference}

Laughlin S, Osorio D (1989) Mechanisms for neural signal enhancement in the blowfly compound eye. J Exp Biol 144:113-146 